\title{
Neoformación en mentón
}

\section{Neoformation in the chin}

\author{
Alberto Ramos-Garibay, ${ }^{*}$ Juan Ramón Trejo-Acuña, ${ }^{\ddagger}$ Larissa Margarita Zárate Flores ${ }^{\S}$
}

\section{CASO CLÍNICO}

Se trata de un paciente del sexo masculino, de 14 años de edad, originario y residente de la Ciudad de México, quien acudió por una dermatosis localizada a cabeza, de la que afectaba cara y de ésta el lado derecho del mentón, constituida por una neoformación oval, eritematoviolácea, de bordes bien delimitados, superficie con fina escama blanquecina seca, con costras sanguíneas en uno de sus extremos, de consistencia firme. De evolución crónica y asintomática (Figura 1).

Al interrogatorio refiere haber iniciado cuatro años atrás con «un granito» en la cara, el cual traumatizó. La lesión fue creciendo lentamente hasta adquirir la morfología actual. Se

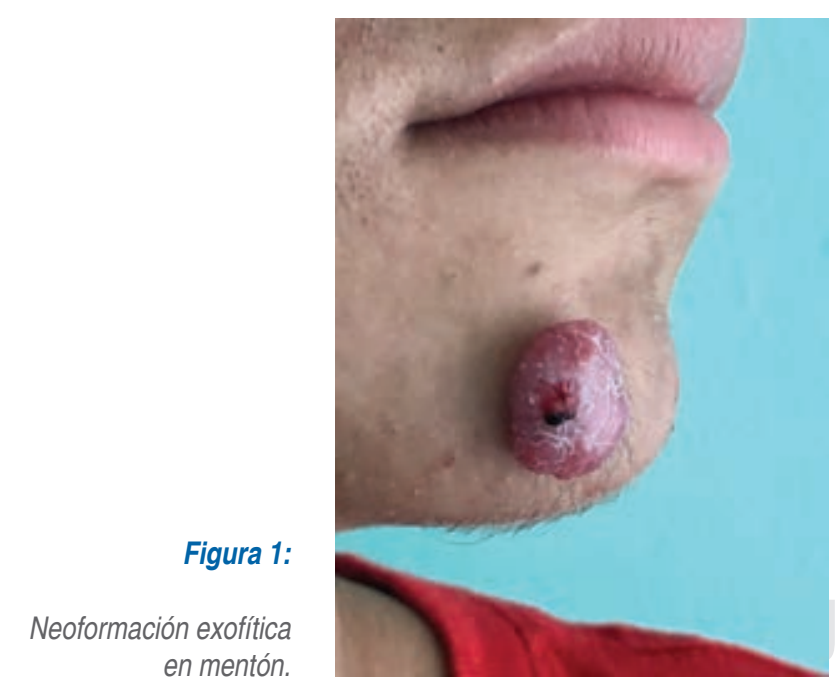

* Subjefe del Servicio de Histopatología.

‡ Dermatólogo.

$\S$ Residente del tercer año de Dermatología.

Centro Dermatológico «Dr. Ladislao de la Pascua», SSCDMX.

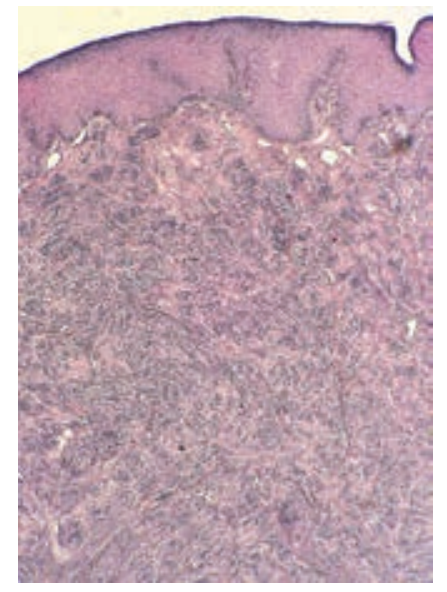

Figura 2:

Epidermis con acantosis irregular, la dermis superficial forma una delgada banda de colágeno normal (H\&E 40x).

Figura 3:

Numerosos agregados

de células

redondas en patrón fusiforme

(H\&E 100x).

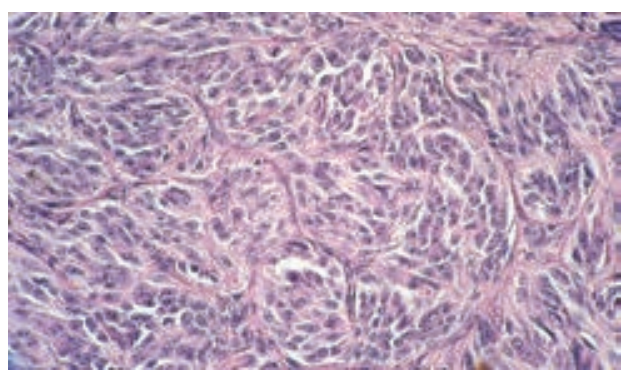

aplicó un ungüento con sulfato de neomicina y retinol, sin mejoría. Se realiza biopsia escisional de la lesión. El estudio histopatológico con tinción de rutina muestra una lesión hemisférica en cuya epidermis predomina la acantosis irregular. La dermis superficial forma una delgada banda de colágeno normal, por debajo de la cual se observan numerosos agregados de células redondas que hacia la profundidad exhiben un patrón fusiforme (Figuras 2 y 3).

Con los datos clínicos e histológicos proporcionados, ¿cuál es su diagnóstico?

Citar como: Ramos-Garibay A, Trejo-Acuña JR, Zárate FLM. Neoformación en mentón. Rev Cent Dermatol Pascua. 2021; 30 (1): 39-42. https://dx.doi.org/10.35366/100591 


\section{DIAGNÓSTICO: NEVO DE SPITZ}

\section{INTRODUCCIÓN}

Sinonimia: Tumor de Spitz, melanoma juvenil, nevo de células epitelioides y fusiformes.

El nevo de Spitz es una neoformación constituida por células epitelioides o células fusiformes. En 1948, Spitz lo describió por primera vez utilizando el término de melanoma juvenil, señalando que se trataba de un tumor melanocítico que se presenta en la niñez, con características clínicas y patológicas similares al melanoma, pero con un comportamiento biológico benigno. ${ }^{1}$ En la literatura mundial, el estudio de este tumor ha sido controversial, debido a su similitud clínica e histopatológica con el melanoma, y a la falta de consenso para establecer criterios diagnósticos o estrategias para manejo clínico. Las neoformaciones melanocíticas con morfología spitzoide se clasifican en tres tipos: nevo de Spitz, tumor de Spitz atípico y melanoma spitzoide. El concepto de tumor de Spitz atípico se refiere a aquellas lesiones histológicas con características atípicas insuficientes para diagnosticar melanoma. ${ }^{2}$

\section{EPIDEMIOLOGÍA}

La incidencia del nevo de Spitz no está bien documentada, pero se calcula entre 1.4 a siete casos por 100,000 personas al año, lo cual representa menos de $1 \%$ de los tumores melanocíticos extirpados en la infancia. El nevo de Spitz aparece durante la infancia o la adolescencia. La probabilidad de que una lesión con apariencia spitzoide se vuelva melanoma incrementa con la edad. No existen diferencias entre géneros, pero es más común en caucásicos. ${ }^{3}$

\section{MANIFESTACIONES CLÍNICAS}

Clínicamente el nevo de Spitz se presenta como una neoformación solitaria, de aspecto papular, cupuliforme, bien delimitada, de consistencia sólida. El color puede variar desde color piel, rosa, rojo o café. Por lo general es rojizo por su alta vascularización y bajo contenido de melanina. Diez por ciento son pigmentados. Su tamaño es menor de 5-6 mm, pero puede llegar a ser de 1-2 $\mathrm{cm}$. Su periodo de crecimiento es rápido (tres a seis meses). Después de la etapa de crecimiento, la lesión se mantiene estática durante años, mostrando una transformación progresiva hasta adquirir el aspecto de un nevo melanocítico, o puede involucionar hasta desaparecer. Puede localizarse en cualquier parte de la superficie corporal, incluyendo mucosas. En niños tiene predilección por la cabeza y el cuello (37\%), mientras que en los adultos jóvenes las lesiones predominan en las extremidades inferiores $(28 \%){ }^{4}$

El diagnóstico puede ser difícil debido a la similitud con otros tumores de la piel, por ello el empleo de la dermatoscopía ha proporcionado una mayor precisión diagnóstica al identificar los diferentes patrones y al proporcionar mayor información sobre su morfología. Ningún patrón es específico del nevo de Spitz, pudiendo estar presentes también en el melanoma, de ahí la importancia de una correcta interpretación.

El nevo de Spitz clásico se ha asociado con la presencia de un patrón vascular compuesto por vasos punteados distribuidos de forma regular sobre un fondo eritematoso, presente en $51 \%$ de los nevos. En la variante pigmentada puede apreciarse un patrón globular o en estallido de estrella, caracterizado por una pigmentación central homogénea con colores que van desde el gris, azul y negro, con proyecciones radiales en la periferia (en el caso del patrón en estallido de estrella), y estructuras ovales o redondas de color café-negro, distribuidas en la periferia en el caso del patrón globular; estos patrones se presentan en 51 y $17 \%$, respectivamente. La despigmentación reticular es otro patrón que inicialmente se asocia con melanoma y que también se observa en el nevo de Spitz, el cual consiste en la formación de líneas que se intersecan alrededor de los glóbulos pigmentados o de vasos sanguíneos. Aunque la dermatoscopía es una técnica útil, no siempre es suficiente para diferenciar entre un nevo de Spitz y un melanoma. La simetría de las estructuras y colores pueden sugerir un nevo de Spitz, mientras que la asimetría o la visualización de crisálidas orienta a considerar un melanoma. ${ }^{5}$

\section{HISTOPATOLOGÍA}

A nivel histopatológico se observa una lesión simétrica, bien delimitada, constituida por células de aspecto epitelioide, fusiformes, o una mezcla de ambas. En general tienen abundante citoplasma y nucléolos centrales prominentes. Los nidos de melanocitos dendríticos intraepiteliales se encuentran orientados perpendicularmente a la epidermis y se pueden ver con frecuencia rodeados por espacios claros. La epidermis puede estar preservada o mostrar hiperplasia regular. El componente dérmico se aprecia maduro en la profundidad, en menor medida en la periferia. Los vasos de la dermis generalmente están dilatados. Una característica común de los nevos de Spitz es la presencia de glóbulos hialinos PAS 
positivos con bordes festoneados (cuerpos de Kamino) en la interfaz dermoepidérmica. Estos cuerpos están compuestos de laminina, colágeno tipo IV y fibronectina y, por lo tanto, no representan cuerpos apoptóticos o citoides. Puede apreciarse un infiltrado inflamatorio linfocítico en la base de la lesión. Se pueden observar mitosis (generalmente $<2 / \mathrm{mm}^{2}$ ) en la parte media o superior de la lesión. ${ }^{6}$ En ocasiones los nevos pueden exhibir atipias que dificultan el diagnóstico.

El estudio inmunohistoquímico es una herramienta complementaria útil en los casos en los que el diagnóstico histopatológico de nevo de Spitz es dudoso, ya que ayuda a diferenciarlo del tumor atípico de Spitz y del melanoma. Es importante recordar que el análisis es subjetivo y depende del criterio del patólogo. Se emplean los siguientes marcadores:

1. MIB1: anticuerpo monoclonal que reacciona con la proteína Ki-67, es una proteína nuclear implicada en la regulación del ciclo celular. Marcador de proliferación expresado en los núcleos del melanoma y tumores atípicos de Spitz; 37\% en melanomas, 10\% en tumores atípicos de Spitz, $5 \%$ en nevo de Spitz, $0.5 \%$ en nevo convencional.

2. HMB45: marcador de mutación con profundidad. Expresado en áreas superficiales con gradiente decreciente hacia la base de la lesión en el nevo de Spitz.

3. p53: menor expresión en nevo de Spitz que en melanoma.

4. p16: regula el ciclo celular, codificando para el gen CDKN2A. Reacción de mayor intensidad en nevo de Spitz que en melanoma, con expresión homogénea.

5. E-cadherina: antígeno expresado de manera difusa en el nevo de Spitz y otras lesiones benignas.

6. CD99: glucoproteína transmembrana expresada más comúnmente en melanoma (56\%) que en nevo de Spitz (5\%).

7. MART1: antígeno de diferenciación melanocítica expresado tanto en nevo de Spitz como en melanoma.

8. S100: débil en nevo de Spitz en comparación con melanoma.

9. S100A6: expresión más intensa y difusa en nevo de Spitz que en melanoma. Patrón en placas o negativo en el nevo de Reed. ${ }^{7}$

\section{DIAGNÓSTICO DIFERENCIAL}

La mayoría de los nevos de Spitz cumplen los criterios histológicos descritos anteriormente, carecen de atipia citológica significativa y su comportamiento biológico es benigno. Dentro de los diagnósticos diferenciales de esta lesión se han detectado varias terminologías imprecisas que incluyen: nevo de Spitz atípico, tumor de Spitz, melanoma de desviación mínima tipo nevo de Spitz y neoplasia melanocítica de potencial maligno indeterminado (tipo nevo de Spitz). ${ }^{8}$ El término nevo de Spitz atípico es el más utilizado; implica un riesgo variable de potencial maligno. Aunque ninguna característica histológica es necesariamente diagnóstica de malignidad, existen algunas potencialmente preocupantes, por ejemplo: la falta de simetría, demarcación lateral deficiente, ulceración, afectación de dermis profunda o grasa subcutánea, maduración deteriorada, diseminación intraepidérmica o pagetoide excesiva, hipercromatismo nuclear, alta relación citoplasmática nuclear, ausencia de cuerpos de Kamino, grandes nidos dérmicos, pigmentación profunda, necrosis, aumento de mitosis dérmicas y mitosis dérmicas atípicas. ${ }^{9}$

El melanoma es uno de los diagnósticos diferenciales más importantes. Para descartarlo es importante solicitar estudios de inmunohistoquímica, ya que todos los nevos de Spitz muestran una positividad S100A6 difusa y fuerte tanto en el componente epidérmico como en el dérmico, mientras que sólo un tercio de los melanomas muestran positividad débil por zonas en el componente dérmico, ausente en el componente epidérmico. ${ }^{7}$

En el melanoma se observa población tumoral aneuploide y en los nevos de Spitz la población de células es diploide. ${ }^{10}$

\section{TRATAMIENTO}

No existe consenso para el manejo de los tumores spitzoides. Se sugiere la extirpación quirúrgica; sin embargo, los nevos de Spitz son completamente benignos. Recientemente, se ha observado que hasta $80 \%$ de los casos evoluciona hacia la desaparición espontánea o conversión a otro tipo más común de nevo melanocítico. En diversos estudios se ha observado que en pacientes $\geq 12$ años de edad, con lesiones que mostraban características spitzoides, el riesgo de melanoma era de $13.3 \%$ y que éste incrementaba con la edad. ${ }^{8}$ Por otro lado, se sugiere dar seguimiento a los pacientes menores de 12 años cada seis meses los 2-3 primeros años, y posteriormente una vez al año, ya que al tener esta postura conservadora se pueden evitar posibles secuelas como cicatrices poco estéticas.

Finalmente, con base en los estudios previos se proponen dos recomendaciones. La primera es para 
aquellas lesiones clínicamente típicas, de aspecto nodular, en las que se sugiere realizar extirpación o monitorización clínica y dermatoscópica a partir de los 15 días después de haber realizado el diagnóstico clínico, y posteriormente cada cuatro $u$ ocho semanas, hasta alcanzar los seis meses sin evidencia de crecimiento, o si se presenta involución. Si se detecta crecimiento en dos ocasiones, se recomienda extirpar. La segunda recomendación es para lesiones típicas de superficie plana. En éstas se sugiere realizar seguimiento cada 2-3 meses, si el aspecto del patrón dermatoscópico es en estallido de estrella, globular o de vasos puntiformes, ya que se consideran no alarmantes; la monitorización debe realizarse durante seis meses en ausencia de crecimiento. ${ }^{10}$

\section{COMENTARIO}

El nevo de Spitz es un tumor muy importante, ya que, aunque su comportamiento biológico es benigno, y asociado con un buen pronóstico, puede diagnosticarse en forma errónea a nivel histopatológico como un melanoma. Desde su descripción, el estudio de esta lesión ha sido motivo de controversia, lo anterior relacionado tanto con el diagnóstico histológico como con la terapéutica más adecuada. El problema se ha hecho considerablemente más complejo debido a la detección de variantes malignas, como el melanoma spitzoide, y por lesiones que se encuentran histológicamente en el límite entre malignidad y benignidad, como el llamado nevo de Spitz atípico. En la actualidad, la recomendación para tratar estas lesiones se basa en la edad del paciente, ya que a mayor edad, mayor probabilidad de atipia o malignidad, por ello se sugiere extirpación completa de la lesión.

En el caso del paciente, motivo de esta publicación, la extirpación se realizó con éxito, en forma completa, con cicatrización adecuada.

\section{REFERENCIAS}

1. Spitz S. Melanomas of childhood. Am J Pathol. 1948; 24: 591-609.

2. Ferrara G, Cavicchini S, Corradin MT. Hypopigmented atypical Spitzoid neoplasms (atypical Spitz nevi, atypical Spitz tumors, Spitzoid melanoma): a clinicopathological update. Dermatol Pract Concept. 2015; 5: 45-52.

3. Herreid PA, Shapiro PE. Age distribution of Spitz nevus vs malignant melanoma. Arch Dermatol. 1996; 132: 352-353.

4. Dika E, Ravaioli GM, Fanti PA, Neri I, Patrizi A. Spitz nevi and other Spitzoid neoplasms in children: overview of incidence data and diagnostic criteria. Pediatr Dermatol. 2017; 34: 25-32.

5. Lallas A, Apalla Z, loannides D, Lazaridou E, Kyrgidis A, Broganelli P et al. Update on dermoscopy of Spitz/Reed naevi and management guidelines by the International Dermoscopy Society. $\mathrm{Br} J$ Dermatol. 2017; 177: 645-655.

6. Kamino H, Misheloff E, Ackerman AB, Flotte TJ, Greco MA. Eosinophilic globules in Spitz's nevi: new findings and a diagnostic sign. Am J Dermatopathol. 1979; 1: 323-324.

7. Sainz-Gaspar L, Sánchez-Bernal J, Noguera-Morel L, HernándezMartín A, Colmenero I, Torrelo A. Nevo de Spitz y otros tumores spitzoides en la infancia. Parte 1: aspectos clínicos, histológicos e inmunohistoquímicos. Actas Dermosifiliogr. 2020; 111: 7-19.

8. Lallas A, Moscarella E, Longo C, Kyrgidis A, de Mestier Y, Vale G et al. Likelihood of finding melanoma when removing a Spitzoid-looking lesion in patients aged 12 years or older. J Am Acad Dermatol. 2015; 72: 47-53.

9. Calonje J, Brenn T, Lazar A, Mckee P. McKee's pathology of the skin. 4ㄹ ed. USA: Elsevier; 2012. p. 1182.

10. Sainz-Gaspar L, Sánchez-Bernal J, Noguera-Morel L, HernándezMartín A, Colmenero I, Torrelo A. Nevo de Spitz y otros tumores spitzoides en la infancia. Parte 2: características citogenéticas y moleculares. Pronóstico y tratamiento. Actas Dermosifiliogr. 2020; 111: 20-25. doi: 10.1016/j.ad.2019.02.010

Correspondencia:

Dr. Alberto Ramos-Garibay

Dr. Vértiz 464, esq. Eje 3 Sur,

Col. Buenos Aires, 06780,

Alcaldía Cuauhtémoc, CDMX.

Tel: 55 5519-6351

E-mail: ramosgari4400@yahoo.com.mx 